\title{
ENTIRE APPROXIMATIONS TO THE TRUNCATED POWERS
}

\author{
FRIEDRICH LITTMANN
}

\begin{abstract}
For a variation diminishing function $g$ which is analytic on a set containing the real line and any real polynomial $P$, we prove that $g+P$ has at most $\operatorname{deg}(P)+2$ real zeros.

Based on this estimate, we present a way to construct entire approximations $G_{n}$ to the truncated powers $x_{+}^{n}$ for $n \in \mathbb{N}_{0}$. Here $x_{+}^{n}=x^{n}$ for $x>0$ and $x_{+}^{n}=0$ for $x<0$. The function $G_{n}$ is constructed in such a way that

$$
G_{n}(x)-x_{+}^{n}=F(x) H_{n}(x)
$$

holds, where $F$ is entire and $H_{n}$ has no zeros on the real line. The function $G_{n}$ can be viewed as an interpolant of $x_{+}^{n}$ with a nodal set that is given by the (real) zeros of $F$.

As an application of this method, we give explicit formulas for best $L^{1}(\mathbb{R})$ - approximation and best one-sided $L^{1}(\mathbb{R})$ - approximation from the class of entire functions with given exponential type $\eta$ to $x_{+}^{n}$. These approximations are given in terms of the logarithmic derivative of the Euler Gamma function.
\end{abstract}

\section{Contents}

1. Introduction

2. Polya-Laguerre Entire Functions 4

3. Variation Diminishing Transforms 6

4. Interpolation by Entire Functions 11

5. Fourier Transforms 15

6. Best $L^{1}(\mathbb{R})$-Approximation from $\mathcal{A}(\eta)$ to $x_{+}^{n} \quad 17$

7. Best One-sided $L^{1}(\mathbb{R})$-Approximation from $\mathcal{A}(\eta)$ to $x_{+}^{n} \quad 20$

References

Date: September 27, 2004.

2000 Mathematics Subject Classification. Primary 42A10; Secondary 41A50.

Key words and phrases. Best $L^{1}(\mathbb{R})$ - approximation, best one-sided approximation, entire interpolation, nodal set, extremal majorants, variation diminishing functions.

The author is supported by a Postdoctoral Fellowship from the Pacific Institute for the Mathematical Sciences (PIMS). 


\section{INTRODUCTION}

Let $n \in \mathbb{N}_{0}$ and define

$$
x_{+}^{n}:= \begin{cases}x^{n} & \text { for } x \geq 0 \\ 0 & \text { for } x<0 .\end{cases}
$$

In particular $x_{+}^{0}=1$ for $x>0, x_{+}^{0}=0$ for $x<0$, and we normalize $x_{+}^{0}$ at the origin by letting $0_{+}^{0}:=1 / 2$.

A non-negative integrable function $g: \mathbb{R} \rightarrow \mathbb{R}$ is said to be variation diminishing if for any bounded continuous $\varphi: \mathbb{R} \rightarrow \mathbb{R}$ the convolution $g * \varphi(x)=\int_{\mathbb{R}} g(t) \varphi(x-t) d t$ has no more changes of sign than $\varphi$ does.

This paper deals with the problem of approximating $x_{+}^{n}$ by an entire function $G_{n}$ such that $G_{n}(x)-x_{+}^{n}$ has sign changes at prescribed real numbers.

The construction presented here extends a method of J. J. Holt and J. D. Vaaler given in Section 2 of [7]. In order to approximate $x_{+}^{n}$, we start with an entire function $F$ which is in the Pólya-Laguerre class (see Definition 2.1 below). By a result of I. J. Schoenberg [17], in any strip $a<\Re z<b$ between two consecutive real zeros $a<b$ of $F$, the function $F$ satisfies

$$
1=F(x) \int_{-\infty}^{\infty} e^{-x t} g(t) d t
$$

for some variation diminishing function $g$ depending on $(a, b)$. We define an approximation to $x_{+}^{n}$ by

$$
G_{n}(x):=F(x) x^{-1} \int_{-\infty}^{0} e^{-x t} g^{(n+1)}(t) d t .
$$

Under certain conditions on $F$, the function $G_{n}$ is entire, and $G_{n}(x)-x_{+}^{n}$ is bounded by constant times $x^{-2}|F(x)|$ for sufficiently large $|x|$ (Theorem 4.3). Moreover, the function $H_{n}$ defined by

$$
G_{n}(x)-x_{+}^{n}=: F(x) H_{n}(x)
$$

has no changes of sign on the real line (Propositions 4.4, 4.5, 4.7). The function $G_{n}$ can be viewed as an entire interpolant to $x_{+}^{n}$ with an a priori prescribed set of interpolating points (or nodal set) that is given by the zeros of $F$.

Let $g$ be a variation diminishing function which is analytic on a set containing the real line, and let $P$ be a polynomial with real coefficients. In Theorem 3.8 we prove the estimate

$$
Z_{\mathbb{R}}[g+P] \leq \operatorname{deg}(P)+2,
$$

where $Z_{\mathbb{R}}[g+P]$ denotes the number of real zeros of $g+P$ counted with multiplicities. Inequality (1.2) is needed to show that $H_{n}$ has no changes of sign on the real line; this is the reason why we restrict $F$ to be the reciprocal of the Laplace transform of a variation diminishing function. 
We say that an entire function $A$ is of exponential type $\eta>0$ if for every $\varepsilon>0$ there exists $C_{\varepsilon}>0$ such that for all $z \in \mathbb{C}$ the inequality

$$
|A(z)| \leq C_{\varepsilon} e^{|z|(\eta+\varepsilon)}
$$

holds. We denote the class of these entire functions by $\mathcal{A}(\eta)$.

We show in Sections 6 and 7 that the unique best $L^{1}(\mathbb{R})$ - approximation from $\mathcal{A}(\eta)$ and the unique best one-sided $L^{1}(\mathbb{R})$ - approximation from $\mathcal{A}(\eta)$ to $x_{+}^{n}$ can be constructed using the interpolation formulas of Section 4 . The formulas obtained below are generalizations of Theorems 4 and 8 in the article [24] of Vaaler. We denote the $j$ th Euler polynomial by $E_{j}$, the $j$ th Bernoulli polynomial by $B_{j}$, and we set $\psi:=\Gamma^{\prime} / \Gamma$, where $\Gamma$ denotes the Euler Gamma function. We define the special functions

$$
\begin{aligned}
\mathfrak{G}_{n, \alpha}(z):=\frac{\sin \pi(z-\alpha)}{\pi} z^{n}\left[\psi\left(2^{-1}(\alpha-z)\right)-\psi(\alpha-z)+\log 2\right. \\
\left.-\frac{1}{2} \sum_{j=0}^{n} E_{j}(\alpha) z^{-j-1}\right], \\
\mathscr{G}_{n, \alpha}(z):=\frac{\sin ^{2} \pi(z-\alpha)}{\pi^{2}} z^{n}\left[\psi^{\prime}(\alpha-z)+\sum_{j=0}^{n} B_{j}(\alpha) z^{-j-1}\right],
\end{aligned}
$$

where $n \in \mathbb{N}_{0}$ and $\alpha \in[0,1]$.

Let $\delta>0$ and $n \in \mathbb{N}_{0}$. Define $\theta_{n}=0$ for even $n$ and $\theta_{n}=1 / 2$ for odd $n$. We show in Section 6 that $\delta^{-n} \mathfrak{G}_{n, \theta_{n}}(\delta z)$ is the unique best approximation in $L^{1}(\mathbb{R})$-norm to $x_{+}^{n}$ from $\mathcal{A}(\pi \delta)$ with $L^{1}$-norm

$$
\int_{\mathbb{R}}\left|x_{+}^{n}-\delta^{-n} \mathfrak{G}_{n, \theta_{n}}(\delta x)\right| d x=\frac{\left|E_{n+1}\left(\theta_{n}\right)\right|}{n+1} \delta^{-n-1} .
$$

The value of the integral in (1.5) can also be obtained from a Markovtype theorem proved by M. G. Krein [10] and B. Sz.-Nagy [22] (see also H. Shapiro [21], Chapter 7). For $n=0$, a different representation of the best approximation was obtained by Vaaler in Theorem 4 of [24].

Let $\delta>0$ and $n \in \mathbb{N}_{0}$. Let $\alpha_{n}$ be a value where the Bernoulli polynomial $B_{n+1}$ assumes a maximum on $[0,1]$ and let $\beta_{n}$ be a value where $B_{n+1}$ assumes a minimum on $[0,1]$. It is shown in Section 7 that $\delta^{-n \mathscr{G}_{n, \alpha_{n}}}(\delta z)$ and $\delta^{-n} \mathscr{G}_{n, \beta_{n}}(\delta z)$ are the unique best one-sided $L^{1}(\mathbb{R})$ - approximations from $\mathcal{A}(2 \pi \delta)$ to $x_{+}^{n}$. The condition 'one-sided' refers to the additional constraint

$$
\delta^{-n} \mathscr{G}_{n, \alpha_{n}}(\delta x) \leq x_{+}^{n} \leq \delta^{-n} \mathscr{G}_{n, \beta_{n}}(\delta x) \text { for } x \in \mathbb{R} .
$$

The $L^{1}$-norms are given by

$$
\begin{aligned}
\int_{-\infty}^{\infty}\left(\delta^{-n} \mathscr{G}_{n, \beta_{n}}(\delta x)-x_{+}^{n}\right) d x & =-\frac{B_{n+1}\left(\beta_{n}\right)}{n+1} \delta^{-n-1}, \\
\int_{-\infty}^{\infty}\left(x_{+}^{n}-\delta^{-n} \mathscr{G}_{n, \alpha_{n}}(\delta x)\right) d x & =\frac{B_{n+1}\left(\alpha_{n}\right)}{n+1} \delta^{-n-1} .
\end{aligned}
$$


For $n=0$, these extremal functions were obtained independently by A. Beurling [3] and A. Selberg (Chapter 20 of [20], compare also the survey [24] by Vaaler). For arbitrary $n$, equations (1.6) and (1.7) were obtained by the author [14] using Euler-Maclaurin summation combined with the Beurling-Selberg approach in the form given by Vaaler in [24].

The result described above can be regarded as an instance where a kind of Markov - type theorem holds, i.e., the solutions to the problems of best $L^{1}(\mathbb{R})$ - approximation and best one-sided $L^{1}(\mathbb{R})$ - approximation from $\mathcal{A}(\eta)$ turn out to be interpolants. Further examples of one-sided approximations with this property can be found in an article of S. W. Graham and Vaaler [5]. For a general connection between interpolants and best approximations see, e.g., Pinkus [16] or Timan [23].

Best one-sided approximations have been of particular interest for certain problems in analytic number theory. These problems include the large sieve inequality (extremal majorants are used in a proof of Selberg [20], see also the survey [24] by Vaaler), a multi-dimensional version of the large sieve (Holt and Vaaler [7]), a quantitative version of the Wiener-Ikehara Tauberian Theorem (Graham and Vaaler [5]), and proofs for Hilbert-type inequalities ([14], Selberg [20], Vaaler [24]).

Most of the material in Sections 2, 3, 4, and 7 appeared previously as part of the thesis [13]. For technical reasons, we approximate $x_{+}^{n}$ rather than $\operatorname{sgn}(x) x^{n}$. However, this is a minor issue, since $2 x_{+}^{n}-\operatorname{sgn}(x) x^{n}=x^{n}$ is an entire function of exponential type 0 .

Acknowledgements. The author would like to thank Harold Diamond for many discussions regarding this subject and the anonymous referees for their helpful comments and suggestions.

\section{Polya-Laguerre Entire Functions}

Definition 2.1. The class $\mathcal{E}$ of Laguerre-Pólya entire functions consists of all entire functions of the form

$$
F(z)=C \exp \left(-c z^{2}+d z\right) z^{\kappa} \prod_{k=1}^{\infty}\left(1-\frac{z}{a_{k}}\right) \exp \left(z / a_{k}\right),
$$

where $c \geq 0, \kappa \in \mathbb{N}_{0}, d, a_{k}(k \in \mathbb{N})$ and $C$ are real, and $\sum_{k=1}^{\infty} a_{k}^{-2}<\infty$. We define

$$
S_{F}:=\{b \in \mathbb{R}: F(b)=0\} \cup\{ \pm \infty\} .
$$

Representation (2.1) shows that any $F \in \mathcal{E}$ is entire, real-valued on the real line, and has only real zeros. A classical theorem by E. Laguerre [11] asserts that the functions in $\mathcal{E}$ are the uniform limits of polynomials having only real roots.

The reciprocals of all elements in $\mathcal{E}$, except the pure exponentials, have representations as Laplace transforms:

Theorem 2.2. Let $F \in \mathcal{E}$ with $F(0) \neq 0$, i.e., $\kappa=0$ in (2.1). 
(a) If $F$ is given by $F(z):=C e^{b z}$ with $b, C \in \mathbb{R}$, then

$$
\frac{1}{F(z)}=C^{-1} e^{-b z}=C^{-1} \int_{-\infty}^{\infty} e^{-t z} \delta_{b}(d t),
$$

where $\delta_{b}$ is the Dirac-measure at $b$.

(b) If $F$ is not of the form $C e^{b z}$ and $F(0) \neq 0$, then there exists an integrable function $g: \mathbb{R} \rightarrow \mathbb{R}$ such that

$$
\frac{1}{F(z)}=\int_{-\infty}^{\infty} e^{-z t} g(t) d t
$$

in a vertical strip containing the origin. The function $g$ has no sign changes and its sign equals the sign of $F(0)$. Moreover,

$$
g(t)=\frac{1}{2 \pi i} \int_{-i \infty}^{i \infty} \frac{e^{z t}}{F(z)} d z
$$

(If $F$ is of the form $C e^{d z}(1-z / a)$ for real values $a, d$ with $a \neq 0$, then (2.4) is understood to be the Cauchy principal value.)

The proof of Theorem 2.2 can be found in [6], Chapters III and IV.

If $a<b$ are two consecutive elements in $S_{F}$ and $c \in(a, b)$, then $F(z-c)^{-1}$ has a representation as a Laplace transform of a function $g$ as in Theorem 2.2 in the strip $a-c<\Re z<b-c$. It follows that the reciprocal of $F$ can be represented as a two-sided Laplace transform in $a<\Re z<b$.

We write $\mathcal{L}[g](z)$ for the two-sided Laplace transform in $(2.3)$.

Lemma 2.3 ([6], II 6.3, III 6.1). Let $F \in \mathcal{E}$, and let $g$ be defined by

$$
\frac{1}{F(z)}=\mathcal{L}[g](z)
$$

in a vertical strip $a<\Re z<b$, where $a$ and $b$ are two consecutive elements in $S_{F}$ defined in (2.2). We have to consider two cases.

(a) Let $F(z)=C e^{d z} P(z)$ where $P$ is a polynomial of degree $n \in \mathbb{N}$ and $d \in \mathbb{R}$. If $n=1$, then $g$ has a discontinuity on the real line. If $n \geq 2$, then $g$ is continuous and $n-2$ times continuously differentiable.

(b) Assume that in (2.1) either $c>0$ or the product is infinite. Then the function $g$ is infinitely differentiable.

We need an estimate of the rate of decay as $t \rightarrow \pm \infty$ of the derivatives introduced in the previous lemma.

Lemma 2.4 (cf. [6], V 2.1). Let $F \in \mathcal{E}$ have at least one zero. Let $a<b$ be two consecutive elements in $S_{F}$ (defined in (2.2)), and let

$$
\frac{1}{F(z)}=\mathcal{L}[g](z) \text { for } a<\Re z<b .
$$


If $a$ and $b$ are both finite, then for any $n \in \mathbb{N}_{0}$ there are polynomials $P_{n}$ and $Q_{n}$ such that

$$
\begin{aligned}
& g^{(n)}(t) \ll\left|P_{n}(t)\right| e^{a t} \text { as } t \rightarrow \infty, \\
& g^{(n)}(t) \ll\left|Q_{n}(t)\right| e^{b t} \text { as } t \rightarrow-\infty .
\end{aligned}
$$

If $a=-\infty$, then $g^{(n)}(t) \ll e^{-K t}$ for all $K>0$ as $t \rightarrow \infty$, and if $b=\infty$, then $g^{(n)}(t) \ll e^{K t}$ for all $K>0$ as $t \rightarrow-\infty$.

In particular if (2.5) is valid in a neighborhood of the origin, $g^{(n)}$ is integrable for any $n \in \mathbb{N}_{0}$.

We need the representations of $\pi \csc \pi(z-\alpha)$ and $\pi^{2} \csc ^{2} \pi(z-\alpha)$ as Laplace transforms. From [6] Chapter III.9 we get for $\alpha \in \mathbb{R}$ that

$$
-\frac{\pi}{\sin \pi(z-\alpha)}=\int_{-\infty}^{\infty} e^{-z t} \frac{e^{\alpha t}}{e^{t}+1} d t \text { for } \alpha-1<\Re z<\alpha
$$

and

$$
\left(\frac{\pi}{\sin \pi(z-\alpha)}\right)^{2}=\int_{-\infty}^{\infty} e^{-z t} \frac{t e^{\alpha t}}{e^{t}-1} d t \text { for } \alpha-1<\Re z<\alpha .
$$

\section{VARiation Diminishing Transforms}

Recall that $\mathcal{L}[g](z)$ denotes the two-sided Laplace transform of $g$. Let $F \in \mathcal{E}$ with $F(0) \neq 0$, and let $g$ be defined by $F(z)^{-1}=\mathcal{L}[g](z)$ in an open strip containing the origin. We will in addition assume that $g$ is analytic on a set containing the real line.

Variation diminishing functions have been investigated in various places in the literature, among them articles by Schoenberg et al. [17], [18], [19], and [4], and books by Hirschman and Widder [6] and by Karlin [9]. We start this section by reviewing results from [6] and [17].

Definition 3.1. Denote by $S^{-}[a(1), \ldots, a(n)]$ the number of changes of sign in the real sequence $a(1), \ldots, a(n)$.

Here zero values do not count as changes of sign. For example, we have $S^{-}[1,0,1]=0$ and $S^{-}[1,0,-1]=1$.

Definition 3.2. If $f(x)$ is a real function defined on $\mathbb{R}$, then

$$
S^{-}[f]:=\sup \left\{S^{-}\left[f\left(x_{1}\right), \ldots, f\left(x_{n}\right)\right] \mid-\infty<x_{1}<\ldots<x_{n}<\infty, n \in \mathbb{N}\right\} .
$$

It is possible that $S^{-}[f]=\infty$.

Definition 3.3. A non-negative, integrable function $g: \mathbb{R} \rightarrow \mathbb{R}$ is said to be variation diminishing if $S^{-}[\varphi * g] \leq S^{-}[\varphi]$ for every bounded continuous function $\varphi: \mathbb{R} \rightarrow \mathbb{R}$.

The following theorem of Schoenberg relates variation diminishing functions and Laplace inverse transforms of reciprocals of elements in $\mathcal{E}$ (cf. [17] and [6], Chapter IV): 
Theorem 3.4. An integrable function $G: \mathbb{R} \rightarrow \mathbb{R}$ is variation diminishing if and only if $G(t)=g(t)$ for almost all $t \in \mathbb{R}$, where $g$ satisfies

$$
F(z)^{-1}=\mathcal{L}[g](z)
$$

in an open strip containing the origin for some $F \in \mathcal{E}$.

Proof. This can be found in IV 2.1 and IV 4.1 of [6].

The following lemma shows that the set of variation diminishing functions is closed with respect to pointwise limits.

Lemma 3.5. Let $\psi,\left(\psi_{k}\right)_{k=1}^{\infty}$ be real functions defined on $\mathbb{R}$, and let $n$ be a non-negative integer. If the condition $\lim _{k \rightarrow \infty} \psi_{k}(t)=\psi(t)$ holds in the pointwise sense, and

$$
S^{-}\left[\psi_{k}\right] \leq n \text { for all } k \in \mathbb{N}
$$

then

$$
S^{-}[\psi] \leq n
$$

Proof. This is Lemma IV.2.1b in [6].

The variation diminishing property is a priori applicable only to bounded continuous functions $\varphi$. The next lemma extends this applicability to all continuous functions $\varphi$ for which the convolution of $\varphi$ with a variation diminishing function exists.

Lemma 3.6. If $g: \mathbb{R} \rightarrow \mathbb{R}$ is a variation diminishing function, $\varphi: \mathbb{R} \rightarrow \mathbb{R}$ is continuous, and $t \mapsto \varphi(t) g(x-t) \in L^{1}(\mathbb{R})$ for all $x \in \mathbb{R}$, then

$$
S^{-}[g * \varphi] \leq S^{-}[\varphi] .
$$

Proof. This is Lemma IV.4.1 in [6].

Let $F \in \mathcal{E}$ and define $g$ by $F(z)^{-1}=\mathcal{L}[g](z)$. By Lemma 2.4, the function $g$ from Theorem 2.2 decreases exponentially fast as $|t| \rightarrow \infty$. Thus, we can apply the variation diminishing property to functions $\varphi$ with subexponential growth on the real line, i.e., $\varphi(t)=e^{o(|x|)}$.

Lemma 2.4 implies in particular that all moments $\int_{\mathbb{R}} t^{k} g(t) d t$ exist and the integral convolution $g * Q(x)$ is defined for every real polynomial $Q$ and every $x \in \mathbb{R}$. We show in the next lemma that this convolution represents a function $P$ which is again a real polynomial.

Lemma 3.7 (cf. [17], Lemma 7). Let $g: \mathbb{R} \rightarrow \mathbb{R}$ be such that all moments

$$
\mu_{k}:=\int_{\mathbb{R}} t^{k} g(t) d t
$$

for $k \in \mathbb{N}_{0}$ exist as Lebesgue integrals and such that $\mu_{0} \neq 0$. Let $P$ be a polynomial of degree $n \in \mathbb{N}_{0}$. There exists a polynomial $Q$ of degree $n$ such that $P=g * Q$. Conversely, for every polynomial $Q$ of degree $n$ there exists a polynomial $P$ of the same degree such that $P=g * Q$.

For fixed $n \in \mathbb{N}_{0}$, the coefficients of $P$ are continuous functions of the coefficients of $Q$, and vice versa. 
Proof. Performing a Taylor expansion of $P$ about $x$ we obtain

$$
\begin{aligned}
Q * g(x) & =\int_{-\infty}^{\infty} g(t) Q(x-t) d t=\sum_{j=0}^{n} \frac{Q^{(j)}(x)}{j !}(-1)^{j} \int_{-\infty}^{\infty} g(t) t^{j} d t \\
& =\sum_{j=0}^{n} \frac{Q^{(j)}(x)(-1)^{j} \mu_{j}}{j !} .
\end{aligned}
$$

Comparing coefficients on both sides shows that the map $Q \mapsto g * Q=P$ sends the coefficients of $Q$ to the coefficients of $P$ via a non-singular matrix transformation.

We are now in a position to establish our estimate of the number of real zeros of $g+P$. Let $S \subseteq \mathbb{C}$, and let $f$ be a function that is analytic on an open set $G \subseteq \mathbb{C}$ containing $S$. Define

$$
Z_{S}[f]:=\text { the number of zeros of } f \text { in } S \text { counted with multiplicity. }
$$

Theorem 3.8. Let $g$ be the Laplace inverse of the reciprocal of some $F \in \mathcal{E}$ in an open strip $S$ containing the origin, and assume that $g$ is analytic in an open set containing the real line. If $P \not \equiv 0$ is a real polynomial and $Q$ is the polynomial defined by $P=g * Q$, then

$$
Z_{\mathbb{R}}[g+P] \leq Z_{\mathbb{R}}[Q]+2 .
$$

In particular $($ since $\operatorname{deg}(P)=\operatorname{deg}(Q)), Z_{\mathbb{R}}[g+P] \leq \operatorname{deg}(P)+2$.

Proof. Suppose first that the real zeros of $g+P$ are all simple. In this case, the number of real zeros equals the number of sign changes of $g+P$. With aid of the Fejér kernel, we shall express $g+P$ as a convolution of $g$ with a function of which we can estimate the number of sign changes. (Compare Chapter IV.5 of [6] for a similar computation.)

Let $K(t)=1-|t|$ for $|t| \leq 1$ and $K(t)=0$ for $|t|>1$. Set $K_{\varepsilon}(t)=$ $1 / \varepsilon \cdot K(t / \varepsilon)$, and recall that $K_{\varepsilon}$ is an approximate identity for $\varepsilon \rightarrow 0+$. We have

$$
g+P=\lim _{\varepsilon \rightarrow 0+} g * K_{\varepsilon}+g * Q=\lim _{\varepsilon \rightarrow 0+} g *\left(K_{\varepsilon}+Q\right) .
$$

The graph of $K_{\varepsilon}$ forms a triangle with base length $2 \varepsilon$ and height $1 / \varepsilon$. For sufficiently small $\varepsilon$, the graph of the polynomial $-Q$ intersects this triangle at most twice. This means that $K_{\varepsilon}+Q$ has at most $S^{-}[Q]+2$ sign changes, namely those sign changes of $Q$ which are not at the origin and the two possible intersections. By Theorem 3.4, the function $g$ is variation diminishing. Thus for all sufficiently small $\varepsilon>0$, equation (3.3) and Lemma 3.5 imply that

$$
Z_{\mathbb{R}}[g+P] \leq S^{-}[g+P] \leq S^{-}\left[K_{\varepsilon}+Q\right] \leq Z_{\mathbb{R}}[Q]+2,
$$

which is the desired conclusion in this case.

Next, suppose that the real zeros of $g+P$ are not all simple. The number of sign changes is now smaller than the number of real zeros, and we cannot 
apply the variation diminishing property directly. We proceed instead by constructing a perturbation of $g+P$.

Lemma 3.9. Let $g$ be as in Theorem 3.8. If $P$ and $Q$ are non-zero real polynomials satisfying $P=g * Q$, then there exist real polynomials $P_{1}$ and $Q_{1}$ with $P_{1}=g * Q_{1}$ such that the inequalities $Z_{\mathbb{R}}[g+P] \leq S^{-}\left[g+P+P_{1}\right]$ and $S^{-}\left[Q+Q_{1}\right] \leq Z_{\mathbb{R}}[Q]$ hold.

An application of (3.4) with $P+P_{1}$ and $Q+Q_{1}$ gives the inequality

$$
Z_{\mathbb{R}}[g+P] \leq S^{-}\left[g+P+P_{1}\right] \leq S^{-}\left[Q+Q_{1}\right]+2 \leq Z_{\mathbb{R}}[Q]+2,
$$

which is the conclusion of Theorem 3.8. To finish the proof of Theorem 3.8, it remains to give the

Proof of Lemma 3.9. The proof will proceed as follows: Change $g+P$ to $g+P+E$ with an error term $E$ in such a fashion that $g+P+E$ has only simple real zeros. We approximate $E$ by a polynomial $P_{1}$, so that Lemma 3.7 is applicable, yielding a polynomial $Q_{1}$ with $P_{1}=g * Q_{1}$. Finally, we investigate the number of real zeros of $Q+Q_{1}$.

Since the Laplace transform of $g$ converges absolutely in a neighborhood of the origin, $g(t)$ decreases exponentially as $t \rightarrow \pm \infty$. Since $g$ is analytic in a region containing the real line and $P$ is a non-zero polynomial, the function $g+P$ has only finitely many real zeros. Let $\left(z_{i}\right)_{i=0}^{n}$ be the list of these zeros such that every zero is repeated according to its multiplicity. We can represent $g+P$ in the form

$$
g(t)+P(t)=\left(t-z_{0}\right)\left(t-z_{1}\right) \ldots\left(t-z_{n}\right) h(t),
$$

where $h$ is a function which is non-zero on $\mathbb{R}$ and analytic on a set containing $\mathbb{R}$. For real numbers $\varepsilon_{i}$, we define a perturbation of $g+P$ by

$$
p_{\varepsilon}(t):=\left(t-z_{0}-\varepsilon_{0}\right) \ldots\left(t-z_{n}-\varepsilon_{n}\right) h(t) .
$$

By choosing $\varepsilon_{i} \neq \varepsilon_{j}$ for $i \neq j$ and letting $\varepsilon_{i} \in(0, a)$ for some sufficiently small $a>0$, we may assume that $p_{\varepsilon}$ has exactly $n+1$ simple zeros (i.e., $n+1$ sign changes) $z_{0}+\varepsilon_{0}, \ldots, z_{n}+\varepsilon_{n}$ on the real line. In order to keep the notation simple, we set $\varepsilon_{i}:=\varepsilon \eta_{i}$ with fixed values $\eta_{i} \in(0,1)$ and a parameter $\varepsilon$ in $(0, a)$. We have

$$
\left(t-z_{0}-\varepsilon \eta_{0}\right) \ldots\left(t-z_{n}-\varepsilon \eta_{n}\right)=\prod_{i}\left(t-z_{i}\right)+\varepsilon L(t, \varepsilon),
$$

where $L(t, \varepsilon)$ is a polynomial in $t$ and in $\varepsilon$.

Set $E_{\varepsilon}(t):=L(t, \varepsilon) h(t)$, where $h$ is defined in (3.5). We obtain from (3.6)

$$
p_{\varepsilon}(t)=g(t)+P(t)+\varepsilon E_{\varepsilon}(t) .
$$

We construct a polynomial $H_{\varepsilon}$ which interpolates $E_{\varepsilon}$ and $E_{\varepsilon}^{\prime}$ at the points $t=z_{i}+\varepsilon_{i}$ and which is of the same sign for large $|t|$ as $P$ is.

Let $H_{1, \varepsilon}$ be a polynomial which interpolates $E_{\varepsilon}$ and $E_{\varepsilon}^{\prime}$ at the points $t=z_{i}+\varepsilon_{i}$ in such a way that the coefficients of $H_{1, \varepsilon}$ are continuous functions 
of $\varepsilon$. Let $m>\operatorname{deg}\left(H_{1, \varepsilon}\right)$ such that $m \equiv \operatorname{deg}(P)$ modulo 2. Let $\sigma \in \mathbb{R} \backslash\{0\}$ be the leading coefficient of $P$ and define a polynomial $H_{\varepsilon}$ by

$$
H_{\varepsilon}(t):=H_{1, \varepsilon}(t)+\sigma t^{m} \prod_{k=0}^{n}\left(t-z_{k}-\varepsilon \eta_{k}\right)^{2} .
$$

By construction, the polynomial $H_{\varepsilon}$ interpolates $E_{\varepsilon}$ and $E_{\varepsilon}^{\prime}$ at the points $t=z_{i}+\varepsilon \eta_{i}$, since the values $H_{\varepsilon}\left(z_{i}+\varepsilon \eta_{i}\right)$ are determined by $H_{1, \varepsilon}$ alone. Therefore, $g+P+\varepsilon H_{\varepsilon}$ has a simple zero at any point $t=z_{i}+\varepsilon_{i}$. Since sign changes occur at these zeros, we obtain

$$
Z_{\mathbb{R}}[g+P] \leq S^{-}\left[g+P+\varepsilon H_{\varepsilon}\right]
$$

for all $\varepsilon \in(0, a)$.

Define $Q_{\varepsilon}$ by $H_{\varepsilon}=g * Q_{\varepsilon}$. By construction, $H_{\varepsilon}$ has leading coefficient $\sigma \neq 0$ for all $\varepsilon \in(0, a)$. By Lemma 3.7, the leading coefficient of $Q_{\varepsilon}$ is therefore non-zero and independent of $\varepsilon$. Thus for all $\varepsilon \in[0, a)$, the polynomial $Q_{\varepsilon}$ is not identically zero. By Hurwitz's theorem, there is a $K>0$ such that for sufficiently small $\varepsilon>0$ all complex zeros of $Q_{\varepsilon}$ are inside the disc $|z| \leq K$, and we may assume that the zeros of $Q$ are also in this disc. Since $Q+\varepsilon Q_{\varepsilon} \rightarrow Q \not \equiv 0$ as $\varepsilon \rightarrow 0$, Hurwitz's theorem applied to a set $S$ containing $[-K, K]$ and all real but no non-real zeros of $Q$ implies that

$$
Z_{[-K, K]}\left[Q+\varepsilon Q_{\varepsilon}\right] \leq Z_{[-K, K]}[Q] .
$$

By the construction of $H_{\varepsilon}$, the degree of $P$ and the degree of $H_{\varepsilon}$ have the same parity and the leading coefficient of $P$ equals the leading coefficient of $H_{\varepsilon}$. Lemma 3.7 implies that the degree of $Q_{\varepsilon}$ and the degree of $Q$ have the same parity and that the leading coefficients of $Q$ and $Q_{\varepsilon}$ are equal. Since for sufficiently small $\varepsilon>0$, the polynomials $Q_{\varepsilon}$ and $Q$ have no real zeros in $|t| \geq K$, we obtain the inequality $\left|Q+\varepsilon Q_{\varepsilon}\right|>|Q|>0$ in $|t| \geq K$, i.e., $Q+\varepsilon Q_{\varepsilon}$ has no real zeros in the region $|t| \geq K$. From (3.8) we obtain

$$
Z_{\mathbb{R}}\left[Q+\varepsilon Q_{\varepsilon}\right] \leq Z_{\mathbb{R}}[Q] .
$$

We set $P_{1}=\varepsilon H_{\varepsilon}$ and $Q_{1}=\varepsilon Q_{\varepsilon}$ for sufficiently small $\varepsilon$. Equations (3.7) and (3.9) complete the proof of Lemma 3.9.

As a corollary of Theorem 3.8, we obtain a bound for the number of real zeros of the tail $g_{(m)}$, where $m \in \mathbb{N}_{0}$.

Corollary 3.10. Let $m \in \mathbb{N}_{0}$, let $g$ be as in Theorem 3.8, and assume $g^{(m)}(0) \neq 0$. Then

$$
g_{(m)}(t)=g(t)-\sum_{k=0}^{m} \frac{g^{(k)}(0)}{k !} t^{k}
$$

has $m+2$ real zeros counted with multiplicity. Depending on the sign of the expression $g^{(m)}(0) g^{(m+1)}(0)$ there are three cases:

(1) If $g^{(m+1)}(0)=0$ then $g_{(m)}$ has a zero of order $m+2$ at the origin and no zeros in $\mathbb{R} \backslash\{0\}$. 
(2) If $g^{(m)}(0) g^{(m+1)}(0)>0$ then $g_{(m)}$ has a zero of order $m+1$ at the origin, precisely one simple zero in $(0, \infty)$, and no zeros in $(-\infty, 0)$.

(3) If $g^{(m)}(0) g^{(m+1)}(0)<0$ then $g_{(m)}$ has a zero of order $m+1$ at the origin, precisely one simple zero in $(-\infty, 0)$, and no zeros in $(0, \infty)$.

Proof. Let

$$
P(t)=-\sum_{k=0}^{m} \frac{g^{(k)}(0)}{k !} t^{k}
$$

Since $g_{(m)}=g+P$, Theorem 3.8 implies $Z_{\mathbb{R}}\left[g_{(m)}\right] \leq m+2$. If $g^{(m+1)}(0)=0$, then $g_{(m)}$ has already a zero of order $m+2$ at the origin, thus $g_{(m)}$ cannot have another zero in $\mathbb{R} \backslash\{0\}$.

If $g^{(m+1)}(0) \neq 0$, then $g_{(m)}$ has a zero of order $m+1$ at the origin, and therefore exactly one zero in $\mathbb{R} \backslash\{0\}$ which has to be simple. Moreover, $g_{(m)}(t) \sim-g^{(m)}(0) t^{m} / m$ ! as $|t| \rightarrow \infty$, because $g$ is converging to zero as $t \rightarrow \pm \infty$ by Lemma 2.4 .

Therefore, the behavior of $g_{(m)}$ at the origin is determined by the sign of $g^{(m+1)}(0)$, and the behavior of $g_{(m)}(t)$ as $t \rightarrow \pm \infty$ is determined by the sign of $-g^{(m)}(0)$.

If $g^{(m+1)}(0)>0$, then $g(t)>0$ for $t>0$ near the origin. If $g^{(m)}(0)>0$, then $g(t)<0$ for large $t$. Therefore, $g$ has a sign change in $(0, \infty)$. Since $g$ has exactly one (simple) zero in $\mathbb{R} \backslash\{0\}$, it has no other zeros in $\mathbb{R} \backslash\{0\}$.

The last case is handled in the same way.

We shall need one additional auxiliary result about variation diminishing functions.

Lemma 3.11. Let $g$ be as in Theorem 3.8, and let $n \in \mathbb{N}_{0}$, then $g^{(n)}$ has exactly $n$ simple real zeros and no multiple real zeros.

Proof. This is IV.5 in [6].

Remark 3.12. If $g$ is analytic on a set containing the real line, and its Laplace transform represents the reciprocal of $F \in \mathcal{E}$ on a strip $a<\Re z<b$ (which does not necessarily include the origin), then $g$ has no real zeros. (This can be seen by applying Lemma 3.11 to $F(z-\theta)$ for some $\theta \in(a, b)$.)

\section{Interpolation by Entire Functions}

In this section we give a generalization of the method of Holt and Vaaler [7] mentioned in the introduction. It is our goal to construct an interpolant $G_{n}$ of $x_{+}^{n}$ with a nodal set given by the zeros of a Pólya-Laguerre entire function $F$. This is accomplished by constructing $G_{n}$ so that

$$
G_{n}(x)-x_{+}^{n}=F(x) H_{n}(x)
$$

holds, where $H_{n}$ is non-zero on the real line.

Recall that $\mathcal{L}[g]$ denotes the two-sided Laplace transform of $g$. 
Definition 4.1. Let $F \in \mathcal{E}$ satisfy $F(z)^{-1}=\mathcal{L}[g](z)$ for $g: \mathbb{R} \rightarrow \mathbb{R}$ in some strip $a<\Re z<b$, where $a$ and $b$ are two consecutive elements in $S_{F}$ (cf. $(2.2))$. If $y \in[a, b]$ and if $g$ is analytic on a set containing the real line, then we call $(F, g)$ a $y$-admissible pair on $(a, b)$.

By equations (2.6) and (2.7), the pairs $\left(-\pi^{-1} \sin \pi(x-\alpha), e^{\alpha t}\left(e^{t}+1\right)^{-1}\right)$ and $\left(\pi^{-2} \sin ^{2} \pi(x-\alpha), e^{\alpha t}\left(e^{t}-1\right)^{-1}\right)$ are 0 -admissible pairs on $(\alpha-1, \alpha)$ for $0 \leq \alpha \leq 1$.

Definition 4.2. Let $(F, g)$ be a 0 -admissible pair on $(a, b)$. For $\Re z<b$ we define

$$
G_{n, F}(z):=\frac{F(z)}{z} \int_{-\infty}^{0} e^{-z t} g^{(n+1)}(t) d t .
$$

We suppress the subscript $F$ and write $G_{n}$ instead of $G_{n, F}$ if no confusion can arise.

Theorem 4.3. Let $(F, g)$ be a 0-admissible pair on $(a, b)$. If at least one of the conditions $F(0)=0$ or $g^{(n)}(0)=0$ is satisfied, then $G_{n}$ has an analytic continuation to the entire complex plane, and the estimate

$$
\left|G_{n}(x)-x_{+}^{n}\right| \ll|x|^{-2}|F(x)|
$$

holds for all real $x$ with $|x| \geq(a+b) / 2$. Moreover,

$$
\left|G_{n}(z)\right| \ll 1+|z|^{n}+|F(z)|
$$

for every $z \in \mathbb{C}$.

Proof. We follow the proof of Lemma 6 in [7]. Using $n+1$ integration by parts, we obtain the representation

$$
z^{n}=\frac{F(z)}{z} \int_{-\infty}^{\infty} g^{(n+1)}(t) e^{-z t} d t
$$

valid in $a<\Re z<b$. After adding and subtracting $z^{n}$, we see that

$$
\begin{aligned}
G_{n}(z) & =z^{n}-\frac{F(z)}{z} \int_{0}^{\infty} g^{(n+1)}(t) e^{-z t} d t \\
& =z^{n}-F(z) \int_{0}^{\infty} g^{(n)}(t) e^{-z t} d t+\frac{F(z)}{z} g^{(n)}(0) .
\end{aligned}
$$

The last equality follows after an integration by parts. By Lemma 2.4, we have $g^{(n+1)}(t) \ll_{\varepsilon} e^{(a+\varepsilon) t}$ for every $\varepsilon>0$ as $t \rightarrow \infty$, so (4.4) and (4.5) are valid in the region $\Re z>a$.

After an integration by parts in $\Re z<b$, we see that

$$
\begin{aligned}
G_{n}(z) & =\frac{F(z)}{z} \int_{-\infty}^{0} g^{(n+1)}(t) e^{-z t} d t \\
& =F(z) \int_{-\infty}^{0} g^{(n)}(t) e^{-z t} d t+\frac{F(z)}{z} g^{(n)}(0) .
\end{aligned}
$$

By assumption, $F(0)=0$ or $g^{(n)}(0)=0$. Thus, the representations (4.5) and (4.7) are analytic in their respective domains even if one of these domains 
contains the origin. Since these halfplanes have the non-empty intersection $a<\Re z<b$, the function $G_{n}$ has an analytic continuation to the entire plane.

To show (4.2), we use the estimates from Lemma 2.4, which imply that

$$
\begin{aligned}
& \left|\int_{0}^{\infty} g^{(n+1)}(t) e^{-z t} d t\right| \ll \int_{0}^{\infty}\left|P_{n}(t)\right| e^{(a-z) t} d t \ll|a-z|^{-1} \text { for } \Re z \geq \frac{a+b}{2}, \\
& \left|\int_{-\infty}^{0} g^{(n+1)}(t) e^{-z t} d t\right| \ll \int_{-\infty}^{0}\left|Q_{n}(t)\right| e^{(b-z) t} d t \ll|b-z|^{-1} \text { for } \Re z \leq \frac{a+b}{2} .
\end{aligned}
$$

Inserting these estimates in (4.4) and (4.6) proves (4.2) and (4.3)

Since $0 \in[a, b]$, equation (4.4) is valid in $\Re z>0$ and (4.6) is valid in $\Re z<0$. Define

$$
H_{n}(z):=\left\{\begin{aligned}
-z^{-1} \int_{0}^{\infty} g^{(n+1)}(t) e^{-z t} d t & \text { for } \Re z>0, \\
z^{-1} \int_{-\infty}^{0} g^{(n+1)}(t) e^{-z t} d t & \text { for } \Re z<0 .
\end{aligned}\right.
$$

We remark that $H_{n}$ depends on $F$ via $g^{(n+1)}$. We obtain from (4.4) and (4.6) the fundamental representation

$$
G_{n}(z)-z_{+}^{n}=F(z) H_{n}(z),
$$

valid for all $z \in \mathbb{C} \backslash\{z: x=0\}$. We shall show that $H_{n}$ is of one sign on the real line. Choosing $F$ appropriately, equation (4.9) enables us to construct approximations $G_{n}$ to $x_{+}^{n}$ with prescribed interpolation points.

First we deal with the case that $n \in\{0,1\}$ and $F$ has a zero at the origin.

Proposition 4.4. Let $(F, g)$ be a 0 -admissible pair on $(a, b)$. If $0 \in\{a, b\}$, then

$$
g^{\prime}(0) H_{0}(x)<0
$$

for all real $x \neq 0$.

Proof. An integration by parts in $F(z)^{-1}=\mathcal{L}[g](z)$ yields

$$
\frac{z}{F(z)}=\int_{-\infty}^{\infty} e^{-z t} g^{\prime}(t) d t \text { for } a<\Re z<b .
$$

Since $z^{-1} F(z)$ is an element of $\mathcal{E}$, Remark 3.12 is applicable, and thus the function $g^{\prime}$ is non-zero on $\mathbb{R}$ (hence does not change sign there). Since $x^{-1} \operatorname{sgn}(x) \geq 0$ for all real $x \neq 0$, inequality (4.10) follows by letting $n=0$ in (4.8).

Proposition 4.5. Let $(F, g)$ be a 0 -admissible pair on $(a, b)$. If $0 \in\{a, b\}$ such that the zero of $F$ at the origin has multiplicity at least two, then

$$
g^{\prime \prime}(0) H_{1}(x)<0
$$

for all real $x \neq 0$. 
Proof. We have $z^{-2} F(z) \in \mathcal{E}$ by assumption. Since $z^{2} F(z)^{-1}=\mathcal{L}\left[g^{\prime \prime}\right](z), g^{\prime \prime}$ has no change of sign by Remark 3.12. Repeating the argument of Proposition 4.4 gives the claim.

We require an auxiliary lemma in order to deal with the remaining cases. Recall the definition

$$
g_{(n)}(t)=g(t)-\sum_{k=0}^{n} \frac{g^{(k)}(0)}{k !} t^{k},
$$

and define for $n \in \mathbb{N}$ an auxiliary function $h_{n}(x, t)$ by

$$
h_{n}(x, t):= \begin{cases}-x^{n} g_{(n-1)}(t) & \text { if } x>0 \text { and } t>0 \\ x^{n} g_{(n-1)}(t) & \text { if } x<0 \text { and } t<0 \\ 0 & \text { if } x t<0\end{cases}
$$

Lemma 4.6. Let $n \in \mathbb{N}$ and let $(F, g)$ be a 0 -admissible pair on $(a, b)$. If $0 \in(a, b)$ and $g^{(n)}(0)=0$, then

$$
g^{(n+1)}(0) \cdot h_{n}(x, t) \leq 0
$$

for all real $x$ and $t$.

Proof. Since $0 \in(a, b)$, the function $g$ is variation diminishing by Theorem 3.4. By Lemma 3.11, the condition $g^{(n)}(0)=0$ implies that $g^{(n-1)}(0) \neq 0$. Thus, Corollary 3.10 is applicable with $m=n-1$. We find that the function $g_{(n-1)}$ has a zero of order $n+1$ at the origin and no other zero in $\mathbb{R}$.

If $n$ is odd, $g_{(n-1)}(t)$ is of one sign for all $t \in \mathbb{R}$ and $x^{n}$ changes its sign at $x=0$. Therefore, $h_{n}(x, t)$ is of one sign for all $x$ and $t$.

If $n$ is even, $g_{(n-1)}$ has a change of sign at the origin and $x^{n}$ is of one sign for all $x \in \mathbb{R}$. Thus, $h_{n}(x, t)$ is of one sign for all $x$ and $t$.

By the definition of $g_{(n-1)}$, the sign of $h_{n}(x, t)$ is determined by the sign of $g^{(n+1)}(0)$.

Proposition 4.7. Let $n \in \mathbb{N}$, and let $(F, g)$ be a 0 -admissible pair on $(a, b)$. If $0 \in\{a, b\}$, assume in addition that $F$ has a zero of order two at the origin. If $g^{(n)}(0)=0$, then

$$
g^{(n+1)}(0) H_{n}(x)<0
$$

for all real $x \neq 0$.

Proof. We have to consider the two cases $0 \in(a, b)$ and $0 \in\{a, b\}$ separately. 
Let $0 \in(a, b)$. Performing $n+1$ integration by parts and using the assumption $g^{(n)}(0)=0$, we obtain the representation

$$
\begin{aligned}
H_{n}(x) & =\left\{\begin{array}{cc}
-x^{n} \int_{0}^{\infty} g_{(n-1)}(t) e^{-x t} d t & \text { for } x>0, \\
x^{n} \int_{-\infty}^{0} g_{(n-1)}(t) e^{-x t} d t & \text { for } x<0
\end{array}\right. \\
& =\int_{-\infty}^{\infty} e^{-x t} h_{n}(x, t) d t \text { for } x \neq 0 .
\end{aligned}
$$

Lemma 4.6 proves that $H_{n}$ has no sign changes. Strict inequality holds because $g_{(n-1)} \not \equiv 0$ is analytic on a set containing the real line.

Assume now $a=0$ or $b=0$. By assumption, the functions $g, g^{\prime}$, and $g^{\prime \prime}$ are analytic on a set containing the real line and represent the reciprocals of $F \in \mathcal{E}, z^{-1} F(z) \in \mathcal{E}$, and $z^{-2} F(z) \in \mathcal{E}$, respectively. Thus, the condition $g^{(n)}(0)=0$ implies $n \geq 3$ by Remark 3.12 .

Since $F$ has a zero of order two at the origin, $z^{-2} F(z)$ has no zero at the origin, and the representation

$$
\frac{z^{2}}{F(z)}=\mathcal{L}\left[g^{\prime \prime}\right](z)
$$

extends beyond $\Re z=0$ to an open strip containing the origin. The condition $g^{(n)}(0)=0$ implies that $\left(g^{\prime \prime}\right)_{(n-2)}=\left(g^{\prime \prime}\right)_{(n-3)}$. The previous argument with $z^{-2} F(z), n-2$, and $g^{\prime \prime}$ instead of $F, n$, and $g$ finishes the proof.

\section{Fourier Transforms}

Recall that $\mathcal{A}(\eta)$ denotes the space of entire functions of finite exponential type $\eta$. This section contains results dealing with the Fourier transform of $A(x)-x_{+}^{n}$ where $A \in \mathcal{A}(\eta)$ and with representations of functions in $\mathcal{A}(\eta)$; these results are needed in Sections 6 and 7 .

For $f \in L^{1}(\mathbb{R})$ we define the Fourier transform of $f$ by

$$
\widehat{f}(t):=\int_{-\infty}^{\infty} f(x) e^{-2 \pi i x t} d x .
$$

Lemma 5.1. Let $n \in \mathbb{N}_{0}$, and let $A \in \mathcal{A}(2 \pi \delta)$ such that $\varphi(x):=A(x)-x_{+}^{n}$ satisfies $\|\varphi\|_{1}<\infty$. The identity

$$
\widehat{\varphi}(t)=-n !(2 \pi i t)^{-n-1}
$$

holds for any $|t| \geq \delta$.

Proof. The argument used to establish Lemma 5.1 uses tempered distributions; we will say below why this is preferable to a proof that uses only $L^{1}(\mathbb{R})$ arguments.

Notation and results about Fourier transforms of distributions of slow growth are taken from the book of L. Hormander [8]. We consider the testing space $\mathcal{S}$ of all functions $\theta: \mathbb{R} \rightarrow \mathbb{C}$ that are infinitely smooth and are such that, as $|t| \rightarrow \infty$, they and all their derivatives decrease to zero 
faster than any power of $|t|^{-1}$. $\mathcal{S}$ is a linear space, and any derivative of a function in $\mathcal{S}$ is again an element of $\mathcal{S}$.

A continuous linear functional on $\mathcal{S}$ is called a tempered distribution. We denote by $\mathcal{S}^{\prime}$ the space of all tempered distributions and we write $u(\theta)$ for the value of $u \in \mathcal{S}^{\prime}$ at $\theta \in \mathcal{S}$. If a functional $u$ in $\mathcal{S}^{\prime}$ is given by $\theta \mapsto u(\theta):=\int_{\mathbb{R}} f(t) \theta(t) d t$ with a function $f: \mathbb{R} \rightarrow \mathbb{C}$, we abuse notation by saying that $f \in \mathcal{S}^{\prime}$.

The Fourier transform $\widehat{u}$ of $u \in \mathcal{S}^{\prime}$ is defined to be the (unique) $\widehat{u} \in \mathcal{S}^{\prime}$ which satisfies

$$
\widehat{u}(\theta)=u(\widehat{\theta}) \text { for all } \theta \in \mathcal{S} .
$$

The assumptions on $\varphi$ imply that $A \in \mathcal{S}^{\prime}$. It follows from the Paley Wiener Theorem for distributions (Theorem 7.3.1 in [8]) that the support of $\widehat{A} \in \mathcal{S}^{\prime}$ is a subset of $[-\delta, \delta]$. The truncated powers $x_{+}^{n}$ are also elements of $\mathcal{S}^{\prime}$. By Example 7.1.17 and equation (3.2.10) of [8], their Fourier transform is a linear combination of derivatives of the Dirac measure at the origin and can be represented by a function anywhere else, namely

$$
\widehat{x_{+}^{n}}(t)=n !(2 \pi i t)^{-n-1} \text { for } t \neq 0 .
$$

Since by assumption $A(x)-x_{+}^{n}$ is integrable, its (continuous) transform is the difference of the respective distributional transforms, which finishes the proof.

The proof of Lemma 5.1 can be given without making use of a distributional argument. This is usually done by proving that the $(n+1)$ st derivative of some explicitly computable approximation $B \in \mathcal{A}(2 \pi \delta)$ to $x_{+}^{n}$ is integrable. Since this derivative is again an element of $\mathcal{A}(2 \pi \delta)$, this leads to

$$
\int_{\mathbb{R}} e^{-2 \pi i x t} d\left(B^{(n)}(x)-x_{+}^{0}\right)=-1 \text { for }|t| \geq \delta,
$$

and Lemma 5.1 follows after $n+1$ integrations by parts and an application of the Paley - Wiener Theorem to $A-B$ for arbitrary $A \in \mathcal{A}(2 \pi \delta)$. The proof of the statement $B^{(n+1)} \in L^{1}(\mathbb{R})$ is technical and leads to a proof of Lemma 5.1 that is longer than the one given here (cf. Section 2 of [24] and the proof of Lemma 2 in [14]). It should be added that these explicit constructions yield representations for $\widehat{\varphi}(t)$ in the range $|t| \leq \delta$ as well, which is desirable for applications.

Remark 5.2. Since the value of the transform of $\varphi(x)=A(x)-x_{+}^{n}$ outside of $(-\delta, \delta)$ does not depend on $A$ at all, any inequality involving values of $\widehat{\varphi}(t)$ with $|t| \geq \delta$ will provide us with a bound that is valid for any $A \in$ $\mathcal{A}(2 \pi \delta)$. The inequalities (6.7) and (7.8), which are used to show that the approximations of Theorem 6.2 and Theorem 7.2 are best approximations, rely on this fact.

We quote two theorems regarding the representation of integrable functions of exponential type $\pi$ or $2 \pi$ in terms of their values at the integers. 
These results are needed to establish uniqueness of the best approximations given in Sections 6 and 7 .

Lemma 5.3. Let $A \in \mathcal{A}(\pi)$ such that its restriction to the real line is in $L^{2}(\mathbb{R})$. The representation

$$
A(z)=\sum_{m=-\infty}^{\infty} A(m) \frac{\sin \pi(z-m)}{\pi(z-m)}
$$

is valid for all $z \in \mathbb{C}$.

Proof. This is (7.19) in Chapter XVI of [25].

By a result of Vaaler [24], the set of integers can also be used to interpolate $A \in \mathcal{A}(2 \pi)$, provided we use the values of $A$ and $A^{\prime}$ at the integers.

Lemma 5.4. Let $A \in \mathcal{A}(2 \pi)$ such that its restriction to the real line is in $L^{2}(\mathbb{R})$. The representation

$$
A(z)=\sum_{m=-\infty}^{\infty} A(m) \frac{\sin ^{2} \pi(z-m)}{\pi^{2}(z-m)^{2}}+\sum_{m=-\infty}^{\infty} A^{\prime}(m) \frac{\sin ^{2} \pi(z-m)}{\pi^{2}(z-m)}
$$

is valid for all $z \in \mathbb{C}$.

Proof. This is a special case of Theorem 9 in [24].

\section{Best $L^{1}(\mathbb{R})$-Approximation from $\mathcal{A}(\eta)$ to $x_{+}^{n}$}

In this section we use Propositions 4.4 and 4.7 to construct approximations in $L^{1}(\mathbb{R})$-norm from $\mathcal{A}(\eta)$ to $x_{+}^{n}$. It turns out that the best $L^{1}(\mathbb{R})$ approximation from $\mathcal{A}(\pi)$ to $x_{+}^{n}$ is an interpolant with a nodal set that is given by a translate of the integers. (This is suggested by inequality (6.7) below.) It turns out that any non-zero element of the nodal set is a simple zero of the difference of the best approximation and $x_{+}^{n}$. This motivates the particular choice of $F=\mathfrak{F}_{\alpha}$ in the next definition.

Let $\alpha \in \mathbb{R}$ and define

$$
\begin{aligned}
\mathfrak{F}_{\alpha}(z) & :=-\pi^{-1} \sin \pi(z-\alpha), \\
\mathfrak{g}_{\alpha}(t) & :=e^{\alpha t}\left(e^{t}+1\right)^{-1}
\end{aligned}
$$

By (2.6), the pair $\left(\mathfrak{F}_{\alpha}, \mathfrak{g}_{\alpha}\right)$ is a 0 -admissible pair on $(\alpha-1, \alpha)$ for $\alpha \in[0,1]$. Definition (4.1) becomes

$$
\mathfrak{G}_{n, \alpha}(z):=\frac{\mathfrak{F}_{\alpha}(z)}{z} \int_{-\infty}^{0} e^{-z t} \mathfrak{g}_{\alpha}^{(n+1)}(t) d t
$$

The function $\mathfrak{G}_{n, \alpha}(z)$ has a representation in terms of the functions $\sin \pi x$, $\psi:=\Gamma^{\prime} / \Gamma$ with the Euler-Gamma function $\Gamma$, and the Euler polynomials $E_{k}$, which can be defined by the generating function identity

$$
\frac{e^{\alpha t}}{e^{t}+1}=\frac{1}{2} \sum_{k=0}^{\infty} E_{k}(\alpha) \frac{t^{k}}{k !}
$$


Proposition 6.1. With $\psi=\Gamma^{\prime} / \Gamma$ we have for $\alpha \in[0,1]$

$$
\begin{aligned}
\mathfrak{G}_{n, \alpha}(z)=\frac{\sin \pi(z-\alpha)}{\pi} z^{n}\left[\psi\left(2^{-1}(\alpha-z)\right)-\right. & \psi(\alpha-z)+\log 2 \\
& \left.-\frac{1}{2} \sum_{j=0}^{n} E_{j}(\alpha) z^{-j-1}\right] .
\end{aligned}
$$

Proof. Let $\Re z<\alpha$. We obtain with $n+1$ integrations by parts

$$
\mathfrak{G}_{n, \alpha}(z)=\mathfrak{F}_{\alpha}(z) z^{n} \int_{0}^{\infty} e^{z t} \mathfrak{g}_{\alpha}(-t) d t+\frac{1}{2} \mathfrak{F}_{\alpha}(z) \sum_{j=0}^{n} E_{j}(\alpha) z^{n-j-1} .
$$

The identity $\left(1+e^{-t}\right)^{-1}=2\left(1-e^{-2 t}\right)^{-1}-\left(1-e^{-t}\right)^{-1}$ implies

$$
-\mathfrak{g}_{\alpha}(-t)=\left(\frac{e^{-2 t}}{t}-\frac{2 e^{-\alpha t}}{1-e^{-2 t}}\right)-\left(\frac{e^{-t}}{t}-\frac{e^{-\alpha t}}{1-e^{-t}}\right)+\left(\frac{e^{-t}}{t}-\frac{e^{-2 t}}{t}\right) .
$$

Equation (6.3) follows for $\Re z<\alpha$ from $\mathfrak{F}_{\alpha}(z)=-\pi^{-1} \sin \pi(z-\alpha)$ and the formulas $\log (b / a)=\int_{0}^{\infty} t^{-1}\left(e^{-a t}-e^{-b t}\right) d t$ for $a>0, b>0$ and $\psi(z)=$ $\int_{0}^{\infty}\left(t^{-1} e^{-t}-e^{-z t}\left(1-e^{-t}\right)^{-1}\right) d t$ for $\Re z>0$, which are recorded in the book of Abramowitz and Stegun [1] as (5.1.32) and (6.3.21). The claim follows for all $z \in \mathbb{C}$ by analytic continuation.

In order to satisfy the assumptions of Propositions 4.4 and 4.7, we have to determine those values of $\alpha$ for which $\mathfrak{g}_{\alpha}^{(n)}(0)=0$. For $k \in \mathbb{N}$, we have

$$
\begin{aligned}
& E_{2 k-1}(1 / 2)=0, \\
& E_{2 k}(0)=E_{2 k}(1)=0 .
\end{aligned}
$$

Moreover, $(-1)^{k} E_{2 k-1}(0)>0$ and $(-1)^{k} E_{2 k}(1 / 2)>0$. The periodic Euler functions can be represented by the Fourier series

$$
\begin{gathered}
E_{2 k}(x-[x])=(-1)^{k} \frac{4(2 k) !}{\pi^{2 k+1}} \sum_{\nu=0}^{\infty} \frac{\sin (2 \nu+1) \pi x}{(2 \nu+1)^{2 k+1}}, \\
E_{2 k-1}(x-[x])=(-1)^{k} \frac{4(2 k-1) !}{\pi^{2 k}} \sum_{\nu=0}^{\infty} \frac{\cos (2 \nu+1) \pi x}{(2 \nu+1)^{2 k}} .
\end{gathered}
$$

These facts can be found in Chapter 2, $\S 2$ of [15]. We define a sequence $\left(\theta_{n}\right)_{n \in \mathbb{N}_{0}}$ by

$$
\theta_{n}:= \begin{cases}0 & \text { if } n \text { is even, } \\ 1 / 2 & \text { if } n \text { is odd }\end{cases}
$$

We normalize our approximations by considering functions of exponential type $\eta=\pi \delta$ with $\delta>0$. 
Theorem 6.2. Let $n \in \mathbb{N}_{0}$ and $\delta>0$. For any entire function $A$ of exponential type $\pi \delta$, the inequality

$$
\int_{-\infty}^{\infty}\left|A(x)-x_{+}^{n}\right| d x \geq \frac{\left|E_{n+1}\left(\theta_{n}\right)\right|}{(n+1) \delta^{n+1}}
$$

holds, with equality if and only if $A(z)=\delta^{-n} \mathfrak{G}_{n, \theta_{n}}(\delta z)$.

Proof. By a change of variable, it is enough to prove the theorem for $\delta=1$. Since $\mathfrak{F}_{0}(z)=-\pi^{-1} \sin \pi z$, we have $\mathfrak{F}_{0}(0)=0$. By Theorem 4.3, the function $\mathfrak{G}_{0,0}$ is entire and $\mathfrak{G}_{0,0}(x)-x_{+}^{0}$ is absolutely integrable. The values $\theta_{n}$ are zeros of $E_{n}$ for $n \geq 1$. Since $\mathfrak{g}_{\alpha}^{(n)}(0)=2^{-1} E_{n}(\alpha)$, the function $\mathfrak{G}_{n, \theta_{n}}$ is entire and the difference $\mathfrak{G}_{n, \theta_{n}}(x)-x_{+}^{n}$ is absolutely integrable in this case as well. Since $\theta_{n} \in[0,1]$, the pair $\left(\mathfrak{F}_{\theta_{n}}, \mathfrak{g}_{\theta_{n}}\right)$ is a 0 -admissible pair on $\left(\theta_{n}-1, \theta_{n}\right)$ for every $n \in \mathbb{N}_{0}$.

Since $\mathfrak{F}_{\theta_{n}}$ is of type $\pi$, the same is true for $\mathfrak{G}_{n, \theta_{n}}$ by (4.3).

Inequality (6.6) is established using a method introduced by J. D. Vaaler in the proof of Theorem 4 of [24]. Let $A$ be any entire function of finite exponential type $\pi$ which satisfies $\left\|A-x_{+}^{n}\right\|_{1}<\infty$, and define

$$
\phi_{n}(x):=A(x)-x_{+}^{n} .
$$

If $n=2 k$ for $k \in \mathbb{N}_{0}$, then

$$
\begin{aligned}
\int_{\mathbb{R}}\left|\phi_{2 k}(x)\right| d x & \geq\left|\int_{\mathbb{R}} \phi_{2 k}(x) \operatorname{sgn} \sin \pi x d x\right| \\
& =\left|\int_{\mathbb{R}} \phi_{2 k}(x) \frac{2}{\pi i} \sum_{\mu \in \mathbb{Z}} \frac{e^{2 \pi i x(\mu+1 / 2)}}{2 \mu+1} d x\right| \\
& =\frac{2}{\pi}\left|\sum_{\mu \in \mathbb{Z}} \frac{1}{2 \mu+1} \widehat{\phi}_{2 k}(-\mu-1 / 2)\right| .
\end{aligned}
$$

We get from Lemma 5.1 that $\widehat{\phi}_{n}(t)=-n !(2 \pi i t)^{-n-1}$ for $|t| \geq 1 / 2$. Equation (6.5) implies the lower bound

$$
\begin{aligned}
\int_{\mathbb{R}}\left|\phi_{2 k}(x)\right| d x & \geq\left|\int_{\mathbb{R}} \phi_{2 k}(x) \operatorname{sgn} \sin \pi x d x\right| \\
& =4 \frac{(2 k) !}{\pi^{2 k+2}} \sum_{\mu=0}^{\infty} \frac{1}{(2 \mu+1)^{2 k+2}}=\frac{\left|E_{2 k+1}(0)\right|}{2 k+1} .
\end{aligned}
$$

Since $\mathfrak{F}_{2 k}(x)=-\pi^{-1} \sin \pi x$ and $(-1)^{k} \mathfrak{g}_{0}^{(2 k+1)}(0)=(-1)^{k} E_{2 k+1}(0)<0$, equation (4.9) and Propositions 4.4 and 4.7 for $k=0$ and $k \geq 1$, respectively, imply the identity

$$
(-1)^{k+1} \operatorname{sgn} \sin \pi x=\operatorname{sgn}\left(\mathfrak{G}_{2 k, 0}(x)-x_{+}^{2 k}\right)
$$

for $k \in \mathbb{N}_{0}$. Thus, we have equality in (6.7) for $A=\mathfrak{G}_{2 k, 0}$.

If we assume that there is equality in (6.7) for some $A \in \mathcal{A}(\pi)$, then $\operatorname{sgn} \sin \pi x\left(A(x)-x_{+}^{2 k}\right)$ does not change sign. Let $k>0$. Since $A$ is continuous, $A(n)=n_{+}^{2 k}=\mathfrak{G}_{2 k, 0}(n)$. By the triangle inequality, $A-\mathfrak{G}_{2 k, 0}$ is 
integrable. We obtain $A=\mathfrak{G}_{2 k, 0}$ from Lemma 5.3. If $k=0$, then a priori $A(n)=\mathfrak{G}_{2 k, 0}(n)$ is known only for integers $n \neq 0$. Since $A(x)-\mathfrak{G}_{0,0}(x)$ is integrable, Lemma 5.3 implies that

$$
A(x)-\mathfrak{G}_{0,0}(x)=\left(A(0)-\mathfrak{G}_{0,0}(0)\right) \frac{\sin \pi x}{\pi x},
$$

which is only integrable if $A(0)=\mathfrak{G}_{0,0}(0)$. Thus, equality follows for $k=0$ as well.

For $n=2 k-1$ with $k \in \mathbb{N}$, the claim follows by considering

$$
\int_{\mathbb{R}}\left|\phi_{2 k-1}(x)\right| d x \geq\left|\int_{\mathbb{R}} \phi_{2 k-1}(x) \operatorname{sgn} \cos \pi x d x\right|
$$

and proceeding as in the previous case.

\section{Best One-Sided $L^{1}(\mathbb{R})$-Approximation from $\mathcal{A}(\eta)$ to $x_{+}^{n}$}

We turn now to the problem of finding extremal one-sided entire approximations of given exponential type to $x_{+}^{n}$. We will use Propositions 4.4, 4.5, and 4.7 in a similar fashion as in the previous section. Now the nodal set for the best one-sided approximation of exponential type $2 \pi$ is a translate of the integers; since we are interested in a one-sided approximation, we require double zeros.

Let $\alpha \in \mathbb{R}$ and define

$$
\begin{aligned}
\mathscr{F}_{\alpha}(z) & :=\pi^{-2} \sin ^{2} \pi(z-\alpha), \\
\gamma_{\alpha}(t) & :=t e^{\alpha t}\left(e^{t}-1\right)^{-1} .
\end{aligned}
$$

The pair $\left(\mathscr{F}_{\alpha}, \gamma_{\alpha}\right)$ is a 0 -admissible pair for $\alpha \in[0,1]$ by $(2.7)$. Definition (4.1) becomes

$$
\mathscr{G}_{n, \alpha}(z):=\frac{\mathscr{F}_{\alpha}(z)}{z} \int_{-\infty}^{0} e^{-z t} \gamma_{\alpha}^{(n+1)}(t) d t
$$

Proposition 7.1. Let $n \in \mathbb{N}_{0}$ and $\alpha \in[0,1]$. With $\psi=\Gamma^{\prime} / \Gamma$ we have

$$
\mathscr{G}_{n, \alpha}(z)=\frac{\sin ^{2} \pi(z-\alpha)}{\pi^{2}} z^{n}\left[\psi^{\prime}(\alpha-z)+\sum_{j=0}^{n} B_{j}(\alpha) z^{-j-1}\right] .
$$

Proof. The lemma follows after $n+1$ integrations by parts with an application of $\psi^{\prime}(z)=\int_{0}^{\infty} t e^{-z t}\left(1-e^{-t}\right)^{-1} d t$, which is formula (6.4.1) in [1].

We have to determine those values of $\alpha$ for which $\gamma_{\alpha}^{(n)}(0)=0$. Since

$$
\frac{t e^{\alpha t}}{e^{t}-1}=\sum_{n=0}^{\infty} B_{n}(\alpha) \frac{t^{k}}{k !} \text { for }|t|<2 \pi
$$

with the Bernoulli polynomials $B_{n}$, we have to consider the zeros of $B_{n}$ in $[0,1]$.

The polynomial $B_{1}$ has its zero at $\alpha=1 / 2$. For odd $n>1$ the zeros of $B_{n}$ are located at $\alpha=0, \alpha=1 / 2$, and $\alpha=1$. For even $n>0$ a general 
formula for the zeros of $B_{n}$ is not known; it is known (cf. [15], Chapter 2 ) that there are two zeros in $[0,1]$ at values $\alpha=z_{n}$ and $\alpha=1-z_{n}$ with $-\pi^{-1} 2^{-2 n-1}<z_{n}-1 / 4<0$, which is an estimate due to D. H. Lehmer [12]. As in [14], we set $z_{0}:=0$ and define for $k \in \mathbb{N}_{0}$

$$
\alpha_{n}:=\left\{\begin{array}{ll}
1-z_{n}, \\
0, \\
z_{n}, \\
1 / 2,
\end{array} \beta_{n}:=\left\{\begin{array}{lll}
z_{n} & \text { if } n \equiv 0 & \bmod 4, \\
1 / 2 & \text { if } n \equiv 1 & \bmod 4, \\
1-z_{n} & \text { if } n \equiv 2 \bmod 4, \\
0 & \text { if } n \equiv 3 \bmod 4 .
\end{array}\right.\right.
$$

The function $B_{n+1}$ assumes a maximum in $[0,1]$ at $\alpha_{n}$ and a minimum in $[0,1]$ at $\beta_{n}$. Note that $\alpha_{0}, \alpha_{1}$, and $\beta_{0}$ are the only values which are not zeros of Bernoulli polynomials. We normalize the approximations in the following theorem by considering functions of exponential type $\eta=2 \pi \delta$ with $\delta>0$.

Theorem 7.2 (cf. [14], Theorem 1). Let $n \in \mathbb{N}_{0}$ and $\delta>0$. The inequality

$$
\delta^{-n} \mathscr{G}_{n, \alpha_{n}}(\delta x) \leq x_{+}^{n} \leq \delta^{-n} \mathscr{G}_{n, \beta_{n}}(\delta x)
$$

holds for all $x \in \mathbb{R}$. Moreover,

(i) for every entire function $A$ of type $2 \pi \delta$ satisfying $A(x) \geq x_{+}^{n}$

$$
\int_{-\infty}^{\infty}\left(A(x)-x_{+}^{n}\right) d x \geq-\frac{B_{n+1}\left(\beta_{n}\right)}{(n+1) \delta^{n+1}}
$$

with equality if and only if $A(x)=\delta^{-n} \mathscr{G}_{n, \beta_{n}}(\delta x)$, and

(ii) for every entire function $A$ of type $2 \pi \delta$ satisfying $A(x) \leq x_{+}^{n}$

$$
\int_{-\infty}^{\infty}\left(x_{+}^{n}-A(x)\right) d x \geq \frac{B_{n+1}\left(\alpha_{n}\right)}{(n+1) \delta^{n+1}}
$$

with equality if and only if $A(x)=\delta^{-n} \mathscr{G}_{n, \alpha_{n}}(\delta x)$.

Proof. A change of variable shows that it is enough to establish the theorem for $\delta=1$. By definition, $\mathscr{F}_{0}(z)=\mathscr{F}_{1}(z)=\pi^{-2} \sin ^{2} \pi z$, therefore $\mathscr{F}_{0}(0)=$ $\mathscr{F}_{1}(0)=0$. As mentioned above, the values $\beta_{n}$ for $n \geq 1$ and $\alpha_{n}$ for $n \geq 2$ are zeros of $B_{n}$. By construction, the equality $\gamma_{\alpha}^{(n)}(0)=B_{n}(\alpha)$ holds. Theorem 4.3 implies that the functions $\mathscr{G}_{n, \alpha_{n}}$ and $\mathscr{G}_{n, \beta_{n}}$ are entire functions. Since $\mathscr{F}_{\alpha}$ is of type $2 \pi$, the same is true for $\mathscr{G}_{n, \alpha_{n}}$ and $\mathscr{G}_{n, \beta_{n}}$ by (4.3).

Since $\alpha_{n}, \beta_{n} \in[0,1]$ for all $n,\left(\mathscr{F}_{\alpha_{n}}, \gamma_{\alpha_{n}}\right)$ and $\left(\mathscr{F}_{\beta_{n}}, \gamma_{\beta_{n}}\right)$ are 0 -admissible pairs.

Since $\gamma_{0}^{\prime}(0)=B_{1}(0)=-1 / 2<0$ and $\gamma_{1}^{\prime}(0)=B_{1}(1)=1 / 2>0$, it follows from Proposition 4.4 that $\mathscr{G}_{0,1}(x) \leq x_{+}^{0} \leq \mathscr{G}_{0,0}(x)$ for all $x \in \mathbb{R}$.

Since $\gamma_{0}^{\prime \prime}(0)=B_{2}(0)=1 / 6>0$, it follows from Proposition 4.5 that $\mathscr{G}_{1,0}(x) \leq x_{+}$for all $x \in \mathbb{R}$, and since $\gamma_{1 / 2}^{\prime \prime}(0)=B_{2}(1 / 2)=-1 / 12<0$, it follows from Proposition 4.7 that $x_{+} \leq \mathscr{G}_{1, \frac{1}{2}}(x)$ for all $x \in \mathbb{R}$.

Let $n \geq 2$. By construction, we have $\gamma_{\alpha_{n}}^{(n+1)}(0)=B_{n+1}\left(\alpha_{n}\right)>0$ and $\gamma_{\beta_{n}}^{(n+1)}(0)=B_{n+1}\left(\beta_{n}\right)<0$. It follows from Proposition 4.7 that $\mathscr{G}_{n, \alpha_{n}}(x) \leq$ $x_{+}^{n} \leq \mathscr{G}_{n, \beta_{n}}(x)$ for all $x \in \mathbb{R}$. 
For the remaining part, we employ a method introduced by Vaaler in the proof of Theorem 8 in [24]. Let $A(x) \geq x_{+}^{n}$ be any entire function of exponential type $2 \pi$ such that $\int_{\mathbb{R}}\left(A(x)-x_{+}^{n}\right) d x<\infty$. Define

$$
\phi_{n}(x)=A(x)-x_{+}^{n} \text {. }
$$

By Lemma 5.1, we have $\widehat{\phi}_{n}(t)=-n !(2 \pi i t)^{-n-1}$ for $|t| \geq 1$. Using Poisson summation and the Fourier series expansion

$$
B_{n}(\alpha)=-\frac{n !}{(2 \pi i)^{n}} \sum_{\ell \neq 0} \frac{e^{2 \pi i \ell \alpha}}{\ell^{n}},
$$

valid for $\alpha \in(0,1)$ and $n \geq 1$, we have for $\alpha \in(0,1)$

$$
\begin{aligned}
0 & \leq \sum_{\nu} \phi_{n}(\alpha+\nu)=\sum_{\ell \in \mathbb{Z}} \widehat{\phi}_{n}(\ell) e^{2 \pi i \ell \alpha}=\widehat{\phi}_{n}(0)-\frac{n !}{(2 \pi i)^{n+1}} \sum_{\ell \neq 0} \frac{e^{2 \pi i \ell \alpha}}{\ell^{n+1}} \\
& =\widehat{\phi}_{n}(0)+\frac{B_{n+1}(\alpha)}{n+1} .
\end{aligned}
$$

It follows that

$$
\int_{\mathbb{R}}\left(A(x)-x_{+}^{n}\right) d x \geq-\inf _{0<\alpha<1} \frac{B_{n+1}(\alpha)}{n+1}=-\frac{B_{n+1}\left(\beta_{n}\right)}{n+1} .
$$

Since $\lim _{y \rightarrow 0+} \mathscr{G}_{n, \beta_{n}}\left(y+\ell+\beta_{n}\right)-\left(y+\ell+\beta_{n}\right)^{n}=0$ for all $\ell \in \mathbb{Z}$, we have equality in (7.8) for $A=\mathscr{G}_{n, \beta_{n}}$. Let $n>0$. By Lemma 5.4, the function $\mathscr{G}_{n, \beta_{n}}$ is the only function for which equality in (7.8) holds. If $n=0$, Lemma 5.4 implies

$$
A(x)-\mathscr{G}_{0,0}(x)=\left(A^{\prime}(0)-\mathscr{G}_{0,0}^{\prime}(0)\right) \frac{\sin ^{2} \pi x}{\pi^{2} x},
$$

and since the right-hand side has to be integrable, we obtain $A^{\prime}(0)=\mathscr{G}_{0,0}^{\prime}(0)$.

Reversing the inequality sign in (7.8) and replacing $\beta_{n}$ by $\alpha_{n}$ yields the statements for $\mathscr{G}_{n, \alpha_{n}}$.

\section{REFERENCES}

[1] M. Abramowitz and I. A. Stegun, Handbook of mathematical functions, National Bureau of Standards, Applied Math. Series 55, U.S. Gov. Printing Office, Washington, D.C. 1964.

[2] N. I. Achieser, Theory of Approximation, Frederick Ungar Publishing Co., New York 1956.

[3] A. Beurling, On functions with a spectral gap, Seminar on Harmonic Analysis, Univ. of Uppsala, March 24, 1942. Published in Collected Works of Arne Beurling: Vol II, Harmonic Analysis, Birkhäuser, Boston, 1989, 359-364.

[4] H. B. Curry and I. J. Schoenberg, On Pólya frequency functions. IV. The fundamental spline functions and their limits, J. Analyse Math. 17, (1966), 71-107.

[5] S. W. Graham and J. D. Vaaler, A class of extremal functions for the Fourier transform, Trans. Amer. Math. Soc. 265 (1981), 283-302.

[6] I. I. Hirschman and D. V. Widder, The Convolution Transform, Princeton University Press 1955, Princeton, New Jersey.

[7] J. J. Holt and J. D. Vaaler, The Beurling-Selberg extremal functions for a ball in Euclidean space, Duke Math. J. 83 (April 1996), No. 1, 203-247. 
[8] L. Hormander, The Analysis of Linear Partial Differential Operators I, SpringerVerlag, New York 1983.

[9] S. Karlin, Total Positivity I, Stanford University Press, Stanford, California, 1968.

[10] M. G. Krein, On the best approximation of continuously differentiable functions on the whole real axis, Dokl. Akad. Nauk SSSR 18 (1938), 615-624. [in Russian]

[11] E. Laguerre, Sur les fonctions du genre zéro et du genre un, Comptes Rendus des Séances de l'Académie des Sciences 98, 828-831.

[12] D. H. Lehmer, On the maxima and minima of Bernoulli polynomials, Amer. Math. Monthly 47 (1940), 533-538.

[13] F. Littmann, Entire Function Majorants, Thesis, University of Illinois at UrbanaChampaign, May 2003.

[14] F. Littmann, Entire Majorants and Euler-Maclaurin summation, submitted.

[15] N. E. Nörlund, Vorlesungen über Differenzenrechnung, Chelsea Publishing Company, New York 1954.

[16] A. Pinkus, On $L^{1}$-approximation, Cambridge tracts in Mathematics Vol. 93, 1989.

[17] I. J. Schoenberg, On Pólya Frequency Functions. I. The totally positive functions and their Laplace transforms. J. Analyse Math. 1, (1951), 331-374, (I. J. Schoenberg, Selected Papers 2, Birkhäuser Verlag 1988.)

[18] I. J. Schoenberg, On Pólya frequency functions. II. Variation-diminishing integral operators of the convolution type, Acta Sci. Math. (Szeged) 12, (1950), 97-106.

[19] I. J. Schoenberg and A. Whitney, On Pólya frequency functions. III. The positivity of translation determinants with an application to the interpolation problem by spline curves, Trans. Amer. Math. Soc. 74, (1953), 246-259.

[20] A. Selberg, Lectures on sieves, Collected Papers II, Springer-Verlag, New York, 1991.

[21] H. Shapiro, Topics in approximation theory, Lecture Notes in Math., vol. 187, Springer Verlag, New York, 1971.

[22] B. Sz.-Nagy, Über gewisse Extremalfragen bei transformierten trigonometrischen Entwicklungen II, Ber. Akad. Wiss. Leipzig (1939).

[23] A. F. Timan, Theory of approximation of functions of a real variable, Pergamon Press, Oxford 1963.

[24] J. D. Vaaler, Some extremal functions in Fourier analysis, Bull. Amer. Math. Soc. 12 (1985), 183-216.

[25] A. Zygmund, Trigonometric series, Vol. II, Cambridge Univ. Press, 1968.

E-mail address: flittman@math.ubc.ca

Department of Mathematics, University of British Columbia, Vancouver BC, V6T 1Z2, CANADA 